\title{
Neurocognitive functioning in schizophrenia and bipolar disorder: clarifying concepts of diagnostic dichotomy vs. continuum
}

\author{
Carissa N. Kuswanto ${ }^{1}$, Min Y. Sum ${ }^{1}$ and Kang Sim ${ }^{1,2 *}$ \\ 1 Research Division, Institute of Mental HealthMoodbridge Hospital, Singapore \\ 2 Department of General Psychiatry, Institute of Mental HealthMoodbridge Hospital, Singapore
}

Edited by:

André Schmidt, University of Basel,

Switzerland

Reviewed by:

Drozdstoy Stoyanov Stoyanov,

Medical University, Bulgaria

Assen Veniaminov Jablensky, The

University of Western Australia,

Australia

Kathryn Eve Lewandowski, McLean Hospital, USA

*Correspondence:

Kang Sim, Department of General

Psychiatry, Institute of Mental

Health/Woodbridge Hospital, 10,

Buangkok View, 539747, Singapore

e-mail: kang_sim@imh.com.sg
The Kraepelinian dichotomy posits that patients with schizophrenia (SCZ) and bipolar disorder (BD) present as two separate psychotic entities such that they differ in terms of clinical severity including neurocognitive functioning. Our study aimed to specifically compare and contrast the level of neurocognitive functioning between SCZ and BD patients and identify predictors of their poor neurocognitive functioning. We hypothesized that patients with SCZ had a similar level of neurocognitive impairment compared with BD. About 49 healthy controls $(\mathrm{HC}), 72 \mathrm{SCZ}$, and $42 \mathrm{BD}$ patients who were matched for age, gender, and premorbid IO were administered the Brief Assessment of Cognition battery (BAC). Severity of psychopathology and socio-occupational functioning were assessed for both patients groups. Both BD and SCZ groups demonstrated similar patterns of neurocognitive deficits across several domains (verbal memory, working memory, semantic fluency, processing speed) compared with $\mathrm{HC}$ subjects. However, no significant difference was found in neurocognitive functioning between BD and SCZ patients, suggesting that both patient groups suffer the same degree of neurocognitive impairment. Patients with lower level of psychosocial functioning $\left[F_{(1,112)}=2.661, p=0.009\right]$ and older age $\left[F_{(1,112)}=-2.625, p=0.010\right]$, not diagnosis or doses of psychotropic medications, predicted poorer overall neurocognitive functioning as measured by the lower BAC composite score. Our findings of comparable neurocognitive impairments between SCZ and BD affirm our hypothesis and support less the Kraepelinian concept of dichotomy but more of a continuum of psychotic spectrum conditions. This should urge clinicians to investigate further the underlying neural basis of these neurocognitive deficits, and be attentive to the associated socio-demographic and clinical profile in order to recognize and optimize early the management of the widespread neurocognitive deficits in patients with SCZ and BD.

Keywords: schizophrenia, bipolar disorder, Kraepelinian dichotomy, neurocognitive functions, psychosocial functioning, psychotic spectrum

\section{INTRODUCTION}

The Kraepelinian dichotomy, a prominent paradigm in psychiatry, has influenced nosology for severe psychiatric disorders such as schizophrenia (SCZ) and bipolar disorder (BD) within diagnostic classification systems including the Diagnostic Statistical Manual of Mental Disorders and the International Classification of Diseases for many decades $(1,2)$. The paradigm posits that patients with dementia praecox (SCZ) and manic depression (BD) present as two separate psychotic disorders $(3,4)$. Evidence in support of the Kraepelinian notion includes volumetric neuroimaging differences in brain regions such as the amygdala, hippocampus, and lateral ventricles that appear to be disorder-specific (5-7). In addition, unlike $\mathrm{BD}$, reduced gray matter volumes and neurocognitive neuromotor impairments occur in the prodromal stage of SCZ despite both having early onset of illness during adolescence and recent findings of common genetic predisposition in $\mathrm{BD}$ and SCZ (6, 8-13). Furthermore, patients with SCZ are thought to suffer more extensive brain morphological abnormalities and more severe neurocognitive deficits in comparison with patients with $\mathrm{BD}$ (14-18). Other than the apparent empirical evidence, the Kraepelinian dichotomy is appealing clinically due to its conceptual and diagnostic simplicity $(19,20)$.

However, several issues challenge the dichotomous paradigm of psychotic disorders with respect to SCZ and BD. These issues include the overlapping organic basis and blurred boundaries between psychoses from emerging biological data $(21,22)$, the non-specificity of psychopathological features such as perceptual disturbances and affective symptoms between SCZ and BD, and the shared genetic susceptibility loci uncovered within recent genome-wide association studies (GWAS) $(23,24)$. Accumulating evidence from clinical observations, antipsychotic treatment, and findings of neurocognitive functioning in SCZ and BD further argued against the Kraepelinian dichotomy. First, clinicians frequently encounter patients who do not fall neatly into either category, such as individuals with schizoaffective disorders and BD patients with prominent psychotic symptoms. Second, dopamine 
dysregulation has been implicated in both disorders, making antipsychotic drugs useful in the management of SCZ as well as $\mathrm{BD}(6,8)$. Third, with regards to neurocognitive functioning, extant studies have reported poorer neurocognitive functioning in patients with SCZ compared to those with BD, but there are also data suggesting that patients with SCZ are comparable with BD patients in terms of neurocognitive impairments $(14,25-31)$. These inconsistent findings on neurocognitive functioning may be due to differences in sample size, specific neurocognitive deficits measured, type of scales used, and presence of other confounders including age and premorbid intelligence (26, 27, 30, 32).

Thus, in this study, we aimed to specifically compare and contrast the level of neurocognitive functioning between patients with SCZ and BD as well as identify predictors of poor neurocognitive functioning in these patients. Based on clinical impression within a tertiary psychiatric hospital context and extant data, we hypothesized that patients with SCZ had a similar level of neurocognitive impairment compared with $\mathrm{BD}$ and the neurocognitive impairment is associated with particular socio-demographic and clinical factors after taking into account premorbid intelligence, level of education, and psychotropic medications prescribed.

\section{MATERIALS AND METHODS \\ PARTICIPANTS}

The cross-sectional study sample comprised of a total of 163 participants [72 patients diagnosed with SCZ, 42 patients with $\mathrm{BD}$, and 49 healthy controls (HC)] who gave their written informed consent to participate in the study after a detailed explanation of the study procedures. Patients with SCZ and BD were recruited from the outpatient settings of the Institute of Mental Health, Singapore. All diagnoses were confirmed by a psychiatrist (Kang Sim) using information obtained from the existing medical record, clinical history, mental status examination, interviews with the patients and their significant others as well as the administration of the Structured Clinical Interview for DSM-IV disorders - Patient Version (SCID-I/P) (33). All patients were maintained on a stable dose of psychotropic medication for at least 2 weeks prior to the recruitment and did not have their medication withdrawn for the purpose of the study. HC were administered the Structured Clinical Interview for DSM-IV disorders - Non-Patient Version (SCID-I/NP) (34). None of the participants had a history of significant and/or unstable/untreated medical illnesses such as seizure disorder, head trauma, or cerebrovascular accidents. Moreover, no subjects had a current or past history of substance use or alcohol use disorder. This study was approved by the Institutional Review Boards of the Institute of Mental Health, Singapore.

\section{NEUROCOGNITIVE ASSESSMENT}

\section{The brief assessment of cognition battery}

The Brief Assessment of Cognition (BAC) battery (35) was administered to all subjects. The subjects were randomly assigned to a sequence of $\mathrm{BAC}$ version $\mathrm{A}$ and $\mathrm{B}$. The $\mathrm{BAC}$ scales include:

1. Verbal memory task - a subject was given 5 attempts to remember 15 words and recall as many words as possible;

2. Digit sequencing task - a subject was presented with clusters of numbers of increasing length and then asked to repeat in order from the lowest to highest length;
3. Token motor task - a subject was given 100 plastic tokens and asked to place 2 tokens at a time within a container as quickly as possible within $60 \mathrm{~s}$;

4. Verbal fluency - divided into semantic and letter fluency, whereby a subject was asked to name as many words as possible within a specific category (e.g., supermarket items), and to name words that begin with a specific letter (e.g., F and S) within 60 s, respectively;

5. Symbol coding task - a subject was asked to write matching numbers from 1 to 9 to symbols within $90 \mathrm{~s}$;

6. Tower of London - as an example, a subject was shown two pictures of three balls of different colors arranged on three different pegs, whereby the balls were arranged differently on each picture and the subjects were asked to give the total number of times the balls in one picture needed to be moved in order to end with the arrangement in the other picture.

We divided the token motor task into two measures, namely the number of tokens correctly placed into a container (tokens correct) and the number of tokens left after $60 \mathrm{~s}$ (tokens left). Although both measures tap motor speed function, tokens correct measure did not credit the subjects for tokens put into container incorrectly (i.e., not picked up and placed at the same time) which required eye-hand coordination and precision in order to perform the task correctly. Digit sequencing task, token motor task, symbol coding, and Tower of London measured working memory, motor speed, attention, and speed of information processing and executive functioning respectively $(35,36)$.

\section{Wide range achievement test (reading scale)}

The wide range achievement test (WRAT) (37) reading subscale was used to measure premorbid intelligence in all the subjects.

\section{CLINICAL ASSESSMENTS \\ Global assessment of functioning}

The global assessment of functioning (GAF) (38) rated (from 0 to 90 ) the severity of symptoms, disability, and the total level of social and occupational functioning. The GAF was administered to both SCZ and BD groups.

\section{Positive and negative syndrome scale}

The positive and negative syndrome scale (PANSS) (39) allowed characterization and quantification of psychotic psychopathology. The scale has 7 items for positive symptoms, 7 items for negative symptoms, and 16 items for general psychopathology. The PANSS was administered to SCZ and BD patients.

\section{Young mania rating scales}

The Young mania rating scales (YMRS) (40) contains 11 items which were used to measure the severity of manic symptoms in BD patients. There are four items that are graded on a $0-8$ scale (irritability, speech, thought content, and disruptive/aggressive behavior). The remaining seven items are graded on a $0-4$ scale. The YMRS scale was administered to SCZ and BD patients.

\section{STATISTICAL ANALYSES}

All statistical tests were performed using PASW for Windows, version 18.0 (SPSS, Inc., Chicago, IL, USA). The normality of distributions of continuous measures was checked with 
the Kolmogorov-Smirnov one-sample test. Socio-demographic variables were compared using two sample student $t$-tests and $F$-test for continuous variables, and chi-square test for categorical variables. Two-way analysis of covariance (ANCOVA) was used to examine for any difference in neurocognitive functioning (BAC task performance) between the groups after adjusting for the covariates described below. We first compared the neurocognitive measures between all three groups, $\mathrm{HC}$ vs. SCZ and $\mathrm{HC}$ vs. $\mathrm{BD}$, and adjusting for covariates of age, gender, years of education, and premorbid IQ. We then specifically compared the BAC item performance between SCZ and BD patients, by adjusting for premorbid IQ, age, gender, years of education, and clinical characteristics such as the duration of illness, duration of untreated psychosis, GAF scores, PANSS composite scores, YMRS scores, and antipsychotic dose in terms of mean daily chlorpromazine (CPZ) mg equivalents. Stepwise linear regression analyses were performed to determine predictors of neurocognitive functioning in patients including socio-demographic (such as age, gender) and clinical characteristics (such as diagnosis, duration of illness, GAF scores, PANSS composite scores, YMRS scores, and antipsychotic dose). The significance level for statistical tests was set at two tailed $p<0.05$.

\section{RESULTS}

\section{SOCIO-DEMOGRAPHIC CHARACTERISTICS}

Across the three subject groups (HC, SCZ, BD), there were significant differences in subject's and mother's years of education but there was no significant difference in age, sex, handedness, and WRAT scores. Compared to patients with $\mathrm{BD}$, patients with SCZ had longer duration of untreated psychosis and lower level of psychosocial functioning as reflected by the lower GAF scores. There was no significant difference in age, gender, handedness, years of education, WRAT scores, age of onset, and duration of illness between SCZ and BD. The socio-demographic and clinical features of the subjects are shown in Table 1.

\section{NEUROCOGNITIVE FUNCTIONING}

Table 2 showed the neurocognitive profile across the entire subject sample based on the BAC battery administration. $\mathrm{HC}$ subjects scored the highest in all BAC items compared to SCZ and BD patients. Compared to HC, patients with SCZ scored lower in BAC tasks of verbal memory, semantic and letter fluency, digit sequencing, tokens left, symbol coding, total BAC score indicating neurocognitive deficits in verbal memory and fluency, working memory, motor speed, and attention. Compared to HC, patients

Table 1 | Demographic and clinical characteristics of the participants

\begin{tabular}{|c|c|c|c|c|c|c|c|c|c|c|}
\hline \multirow[t]{3}{*}{ Characteristic } & \multicolumn{2}{|c|}{$\mathrm{HC}(n=49)$} & \multicolumn{2}{|c|}{$\operatorname{scZ}(n=72)$} & \multicolumn{2}{|c|}{$\mathrm{BD}(n=42)$} & \multicolumn{4}{|c|}{ Statistical analysis } \\
\hline & \multirow[b]{2}{*}{ Mean } & \multirow[b]{2}{*}{ SD } & \multirow[b]{2}{*}{ Mean } & \multirow[b]{2}{*}{ SD } & \multirow[b]{2}{*}{ Mean } & \multirow[b]{2}{*}{ SD } & \multicolumn{2}{|c|}{ SCZ vs. BD } & \multicolumn{2}{|c|}{ All three groups } \\
\hline & & & & & & & $t$-test & $p$ & F-test & $\boldsymbol{p}$ \\
\hline Age & 31.9 & 10.4 & 31.47 & 8.29 & 32.57 & 9.673 & -0.642 & 0.522 & 0.191 & 0.827 \\
\hline Subject's years of education & 13.59 & 1.62 & 12.31 & 2.039 & 12.15 & 2.291 & 0.364 & 0.717 & 7.849 & $0.001 *$ \\
\hline Father's years of education & 8.53 & 4.22 & 7.62 & 3.697 & 7.31 & 3.317 & 0.447 & 0.655 & 1.454 & 0.237 \\
\hline Mother's years of education & 7.88 & 4.29 & 6.93 & 3.815 & 4.98 & 4.129 & 2.551 & $0.012^{*}$ & 6.228 & $0.002^{*}$ \\
\hline WRAT scores & 49.78 & 4.25 & 49.75 & 5.301 & 49.88 & 4.994 & -0.13 & 0.897 & 0.75 & 0.474 \\
\hline Duration of illness & - & - & 4.77 & 5.317 & 3.32 & 56.39 & 1.394 & 0.166 & - & - \\
\hline Age of onset & - & - & 26.69 & 8.073 & 28.24 & 9.294 & -0.931 & 0.354 & - & - \\
\hline Duration of untreated psychosis & - & - & 1.715 & 2.206 & 0.296 & 0.528 & 4.094 & $<0.001^{*}$ & - & - \\
\hline Mean daily CPZ mg equivalents & - & - & 185.76 & 197.47 & 157.38 & 174.89 & 0.771 & 0.442 & - & - \\
\hline \multicolumn{11}{|l|}{ GAF scores } \\
\hline Total & - & - & 50.18 & 18.821 & 64.9 & 21.101 & -3.852 & $<0.001 *$ & - & - \\
\hline Symptoms & - & - & 54.36 & 20.292 & 64.98 & 21.39 & -2.641 & $0.009^{*}$ & - & - \\
\hline Disability & - & - & 51.46 & 19.092 & 65.69 & 19.749 & -3.791 & $<0.001^{*}$ & - & - \\
\hline \multicolumn{11}{|l|}{ PANSS } \\
\hline Positive symptoms & - & - & 10.79 & 3.715 & 8.55 & 2.839 & 3.379 & $0.001^{*}$ & - & - \\
\hline Negative symptoms & - & - & 8.33 & 2.116 & 7.05 & 0.309 & 3.906 & $<0.001 *$ & - & - \\
\hline General psychopathology & - & - & 20.89 & 3.71 & 17.79 & 1.828 & 5.067 & $<0.001^{*}$ & - & - \\
\hline Total score & - & - & 40.01 & 7.135 & 33.38 & 4.120 & 5.506 & $<0.001 *$ & - & - \\
\hline \multirow[t]{2}{*}{ YMRS } & - & - & 1.56 & 2.469 & 3.4 & 4.607 & 2.485 & $0.015^{*}$ & - & - \\
\hline & $N$ & $\%$ & $N$ & $\%$ & $N$ & $\%$ & $x^{2}$ & $p$ & $x^{2}$ & $p$ \\
\hline Male & 28 & 57.1 & 38 & 52.8 & 17 & 40.5 & 1.608 & 0.205 & 2.442 & 0.295 \\
\hline Right-handed & 46 & 93.9 & 70 & 97.2 & 40 & 97.6 & 1.148 & 0.284 & 1.102 & 0.576 \\
\hline
\end{tabular}

BD, bipolar disorder; CPZ, chlorpromazine; GAF, global assessment of functioning; HC, healthy controls; PANSS, positive and negative syndrome scale; SCZ, schizophrenia; WRAT, wide range achievement test; YMRS, young mania rating scale. ${ }^{*} p<0.05$. 
Table 2 | Neurocognitive functioning across subjects based on brief assessment of cognition battery (BAC).

\begin{tabular}{|c|c|c|c|c|c|c|c|c|c|c|c|c|c|c|}
\hline BAC domains & \multicolumn{2}{|c|}{ HC $(n=49)$} & \multicolumn{2}{|c|}{$\operatorname{SCZ}(n=72)$} & \multicolumn{2}{|c|}{$\mathrm{BD}(n=42)$} & \multicolumn{6}{|c|}{ ANCOVA $^{a}$} & \multicolumn{2}{|c|}{$\frac{\text { ANCOVA }^{b}}{\text { SCZ vs. BD }}$} \\
\hline Verbal memory & 9.07 & 1.92 & 7.60 & 2.11 & 7.85 & 1.91 & 6.45 & $0.002 * *$ & 9.84 & $0.002 * *$ & 11.99 & $0.001 * *$ & 0.92 & 0.340 \\
\hline Digit sequencing & 21.62 & 3.01 & 19.04 & 4.54 & 18.68 & 4.07 & 4.64 & $0.011^{*}$ & 7.13 & $0.009 *$ & 9.74 & $0.002^{* *}$ & 0.05 & 0.942 \\
\hline Semantic fluency & 24.64 & 5.82 & 20.10 & 5.77 & 21.17 & 5.86 & 6.13 & $0.003^{* *}$ & 12.05 & $0.001 * *$ & 5.58 & $0.020^{*}$ & 0.18 & 0.674 \\
\hline Letter fluency & 13.91 & 3.71 & 12.51 & 4.51 & 12.21 & 4.82 & 4.27 & $0.016^{*}$ & 6.83 & $0.010^{*}$ & 7.35 & $0.008^{*}$ & 0.02 & 0.895 \\
\hline Symbol coding & 64.68 & 10.43 & 49.21 & 11.42 & 50.55 & 10.45 & 27.47 & $<0.001 * *$ & 48.31 & $<0.001^{* *}$ & 32.85 & $<0.001^{* *}$ & 0.08 & 0.773 \\
\hline Tower of London & 17.04 & 2.12 & 16.17 & 3.79 & 16.27 & 3.49 & 0.24 & 0.785 & 0.36 & 0.548 & 0.17 & 0.683 & 0.08 & 0.783 \\
\hline BAC total & 277.66 & 28.25 & 239.36 & 77.12 & 232.60 & 43.69 & 6.27 & $0.002 * *$ & 8.00 & $0.006^{*}$ & 28.55 & $<0.001^{* *}$ & 0.36 & 0.552 \\
\hline
\end{tabular}

$B A C$, brief assessment of cognition battery; $B D$, bipolar disorder; $C P Z$, chlorpromazine; GAF, global assessment of functioning; $H C$, healthy controls; $S C Z$, schizophrenia; WRAT, wide range achievement test. ${ }^{*} p<0.05{ }^{*} p<0.01$.

${ }^{a}$ Adjusted for WRAT, age, gender, and years of education.

${ }^{b}$ Adjusted for WRAT, age, gender, years of education, duration of untreated psychosis, duration of illness, GAF (total, symptoms, and disability), antipsychotic dose, and PANSS and YMRS composite scores.

with BD scored lower in BAC tasks of verbal memory, semantic and letter fluency, digit sequencing, tokens correct, symbol coding, total BAC score indicating almost similar neurocognitive deficits as SCZ in verbal memory and fluency, working memory, motor speed, and attention. After taking into account multiple comparisons (at conservative threshold of $p<0.005$ ), patients with SCZ scored lower on tasks of verbal memory, semantic fluency, symbol coding, and patients with BD scored lower on verbal memory, digit sequencing, and symbol coding when compared with HC.

However, there was no significant difference in all neurocognitive domains of BAC when SCZ and BD groups were compared and after controlling for covariates such as demographic characteristics (age, gender, years of education, WRAT scores) and clinical features (duration of untreated psychosis, antipsychotic dose, as well as GAF, PANSS, and YMRS composite scores). We also noted the possibility that a history of psychosis in $\mathrm{BD}$ patients may be associated with poorer performance in neurocognitive tasks compared to those BD subjects without history of psychosis (30). Thus, we performed a post hoc analysis whereby we compared BD patients with history of psychosis and those without and we found no significant difference in all neurocognitive performance in all BAC domains.

Multivariate linear regression analyses with the overall BAC score as the dependent variable and demographic (age, gender, education) and clinical factors (diagnosis, WRAT scores, GAF scores, PANSS composite scores, YMRS scores, duration of illness, duration of untreated psychosis, antipsychotic dose) as predictor variables revealed that patients with lower level of psychosocial functioning as indicated by lower GAF score $\left[F_{(1,112)}=2.023\right.$, $p=0.046]$ and older age $\left[F_{(1,112)}=-2.190, p=0.031\right]$, but not diagnosis or doses of psychotropic medications, were associated with poorer overall neurocognitive functioning as measured by the lower BAC composite score.

\section{DISCUSSION}

There were several main findings in this study. First, patients with $\mathrm{BD}$ and $\mathrm{SCZ}$ were found to have greater neurocognitive impairments in most of the BAC domains compared to the $\mathrm{HC}$ who were matched for age, gender and premorbid intelligence. Second, the extensive neurocognitive impairments found in SCZ were comparable with those observed in patients with $\mathrm{BD}$. Third, the level of psychosocial functioning and age of patients with $\mathrm{SCZ}$ and $\mathrm{BD}$ were associated with neurocognitive impairment and not diagnosis or antipsychotic dose. Our findings highlighted that both SCZ and BD patients in our study sample suffered largely similar neurocognitive impairments, which support a continuum concept of psychosis rather than the Kraepelinean concept of dichotomous psychotic conditions.

Our findings of comparable and extensive neurocognitive impairments in both SCZ and BD are consistent with some earlier studies reporting findings of specific neurocognitive impairments across the patient groups. For example, BD and SCZ patients scored significantly worse than control subjects in verbal memory task (assessed by the California Verbal Learning Test), which did not differ when both patient groups were compared $(25,29)$. Likewise, motor speed was also impaired in patients with SCZ/BD during the Grooved Pegboard test when compared with HC (14, 31). Furthermore, other studies have shown comparable impairments in domains of verbal fluency and working memory within both SCZ and BD patients $(14,25,28)$. In terms of executive functioning, a number of studies found comparable neurocognitive deficits in both patient groups irrespective of the tests used such as Wisconsin Card Sorting Test (WCST) $(14,29,41)$, Trail Making Test B (TMT B) $(28,31)$, and Tower of Hanoi (29). Overall, our findings add to the literature to support the notion of comparable and pervasive neurocognitive impairments in patients with $\mathrm{BD}$ and SCZ $(17,29)$. 
What may be the biological factors that underlie SCZ and BD which may be relevant to the observed neurocognitive impairments? First, recent evidence from large-scale GWAS has pointed to possible common susceptibility genes underlying SCZ and BD which may affect neurocognitive functioning. These common vulnerability cross disorder genes include ZNF804A, CACNA1C, NRGN, and PBRM1 (5, 19, 23, 24, 42-45). A recent study from the Psychiatric Genome Consortium reported that there are 14 genetic loci associated with both SCZ and BD (46), and shared genetic effects between SCZ and BD accounted for $52 \%$ of the genetic variance in SCZ and 69\% for BD in the Swedish population (44). Second, neuroimaging aberrations of brain morphology and function have been noted. For example, voxel-based morphometry studies found that there was a substantial overlap in gray matter volumetric reductions such as the prefrontal cortex, thalamus, caudate, medial temporal lobe, insula, and the anterior cingulate regions in SCZ and BD $(7,15,47)$. Third, while genetic risks for SCZ and $\mathrm{BD}$ are associated with different gray matter changes, McDonald et al. (48) suggested that they share white matter endophenotypes with the reduction of white matter volume in the left frontal and temporoparietal regions in both SCZ and $\mathrm{BD}$.

Of note, older age and poorer psychosocial functioning were found to be associated with neurocognitive impairments in our patients. These findings are in line with that of other studies whereby older age was associated with decreased gray matter volume (49) and poorer neurocognitive performance in SCZ, and which can affect psychosocial functioning (50). The lifetime history of psychotic features in BD patients has also been found to worsen subsequent neurocognitive performance such as cognitive flexibility and working memory indicating age related effects on neurocognitive functioning in $\mathrm{BD}(10,26,27,30,32,51)$. However, in contrast, Gildengers et al. (52) found no difference in the rate of neurocognitive decline between $\mathrm{BD}$ and healthy subjects with age.

We found that antipsychotic medication dose was not associated with neurocognitive impairment in SCZ and BD in this study although there is evidence of antipsychotic medications affecting changes in brain structures and neurocognitive performance. Previous studies have reported both improvements in neurocognitive impairments with antipsychotic treatment in SCZ and BD (26) but negative impact on cognition have also been reported in BD $(53,54)$. In SCZ patients, some studies reported that the administration of typical antipsychotic medication provides only modest-to-moderate gains in multiple cognitive domains $(55,56)$, while other studies found no correlation between antipsychotic medication dose and neurocognitive functioning in BD and SCZ $(9,18,57)$.

Recent data also suggest that neurocognitive deficits may potentially serve as endophenotypical trait markers for BD and SCZ (58-60). Unlike a state marker whereby neurocognitive performance may vary with and be influenced by current clinical states such as affective change and psychotic phenomenology, a trait marker remains stable throughout the illness course and may be related to genetic factors $(60,61)$. For SCZ, a twin study by Pardo et al. (58) noted preservative errors on the WCST not only in the SCZ twin but also within non-SCZ monozygotic co-twin suggesting a spectrum of genetic liability. Furthermore, clinical symptoms do not seem to play a role in worsening cognitive performance in ultra-high risk of psychosis subjects (62). In BD, Sobzcak et al. (59) found deficits in memory function, psychomotor performance and attention in high risk relatives suggesting potential neurocognitive trait markers for BD. In a recent longitudinal study involving $\mathrm{BD}$ patients, the severity of depressive symptoms did not predict change of performance in any cognitive domains and all domains remained stable over the course of 6 years (60).

There are several limitations to the study. First, our sample size was rather small and the study solely recruited patients with remitted SCZ and BD seen at the outpatient setting of a tertiary psychiatric hospital and thus the results may not be generalizable to patients within the inpatient and in non-tertiary psychiatric settings. Second, this was a cross-sectional study and did not allow observations of changes of neurocognitive functioning over the course of time. Third, the administrators of the neurocognitive tests were not blinded to the diagnoses of the subjects. Lastly, we did not examine other biological correlates such as genetic factors, neurophysiological measures but we intend to do so as a follow up study of these findings. These multimodality studies can potentially elucidate biological underpinnings or correlates of these neurocognitive impairments in SCZ and BD.

In conclusion, we found that patients with BD and SCZ suffer widespread neurocognitive deficits involving almost all the examined cognitive domains. The severity of neurocognitive deficits has been found to be significantly associated with various factors such as the level of psychosocial functioning and age but not specific diagnoses or antipsychotic medications. The underlying biological basis of these neurocognitive deficits may be related to factors common to both conditions and awaits better definition. Our findings support the dimensional concept of psychotic spectrum conditions along a continuum rather than the dichotomous concept as proposed by Kraepelin. Although DSM-V (63) still retains the existing nosological boundaries between BD and SCZ, clinicians would have to remain vigilant in managing each case of SCZ or BD by taking into account their clinical, socio-demographic factors, and assessing for the presence of specific neurocognitive impairment so as to better optimize the care and cater for more individually tailored treatment plans for these patients with potentially crippling conditions.

\section{ACKNOWLEDGMENTS}

This study was supported by NHG SIG (12003/12004/11003) research grants awarded to Dr. Kang Sim. We would like to thank all of the patients, their families, and our hospital staff for their support of this study.

\section{REFERENCES}

1. Möller HJ. Systematic of psychiatric disorders between categorical and dimensional approaches: Kraepelin's dichotomy and beyond. Eur Arch Psychiatry Clin Neurosci (2008) 258(Suppl 2):48-73. doi:10.1007/s00406-008-2004-3

2. Sorias S. Is the neo-Kraepelinian paradigm in a phase of crisis? Turk Psikiyatri Derg (2012) 23:117-29.

3. Berrios GE, Beer D. The notion of a unitary psychosis: a conceptual history. Hist Psychiatry (1994) 5:13-36. doi:10.1177/0957154X9400501702

4. Kraepelin E. Dementia Praecox and Paraphenia. Edinburg: Livingstone (1919).

5. Bellivier F, Geoffroy PA, Scott J, Schurhoff F, Leboyer M, Etain B. Biomarkers of bipolar disorder: specific or shared with schizophrenia? Front Biosci (Elite Ed) (2013) E5:845-63. doi:10.2741/E665 
6. Murray RM, Sham P, Van Os J, Zanelli J, Cannon M, McDonald C. A developmental model for similarities and dissimilarities between schizophrenia and bipolar disorder. Schizophr Res (2004) 71:405-16. doi:10.1016/j.schres.2004.03.002

7. Yu K, Cheung C, Leung M, Li Q, Chua S, McAlonan G. Are bipolar disorder and schizophrenia neuroanatomically distinct? An anatomical likelihood metaanalysis. Front Hum Neurosci (2010) 4:189. doi:10.3389/fnhum.2010.00189

8. Demjaha A, MacCabe JH, Murray RM. How genes and environmental factors determine the different neurodevelopmental trajectories of schizophrenia and bipolar disorder. Schizophr Bull (2012) 38:209-14. doi:10.1093/schbul/sbr100

9. Fusar-Poli P, Deste G, Smieskova R, Barlati S, Yung AR, Howes O, et al. Cognitive functioning in prodromal psychosis: a meta-analysis. Arch Gen Psychiatry (2012) 69:562-71. doi:10.1001/archgenpsychiatry.2011.1592

10. Lewandowski KE, Cohen BM, Öngur D. Evolution of neuropsychological dysfunction during the course of schizophrenia and bipolar disorder. Psychol Med (2011) 41:225-41. doi:10.1017/S0033291710001042

11. Napal O, Ojeda N, Sánchez P, Elizagárate E, Peña J, Ezcurra J, et al. The course of the schizophrenia and its impact on cognition: a review of literature. Actas Esp Psiquiatr (2012) 40:198-220.

12. O'Donnell BF. Cognitive impairment in schizophrenia: a life span perspective. Am J Alzheimers Dis Other Demen (2007) 22:398-405. doi:10.1177/ 1533317507304745

13. Young RC, Murphy CF, Heo M, Schulberg HC, Alexopoulos GS. Cognitive impairment in bipolar disorder in old age: literature review and findings in manic patients. J Affect Disord (2006) 92:125-31. doi:10.1016/j.jad.2005.12.042

14. Depp CA, Moore DJ, Sitzer D, Palmer BW, Eyler LT, Roesch S, et al. Neurocognitive impairment in middle-aged and older adults with bipolar disorder: comparison to schizophrenia and normal comparison subjects. J Affect Disord (2007) 101:201-9. doi:10.1016/j.jad.2006.11.022

15. Ellison-Wright I, Bullmore E. Anatomy of bipolar disorder and schizophrenia: a meta-analysis. Schizophr Res (2010) 117:1-12. doi:10.1016/j.schres.2009.12.022

16. Krabbendam L, Arts B, van Os J, Aleman A. Cognitive functioning in patients with schizophrenia and bipolar disorder: a quantitative review. Schizophr Res (2005) 80:137-49. doi:10.1016/j.schres.2005.08.004

17. Schretlen DJ, Cascella NG, Meyer SM, Kingery LR, Testa SM, Munro CA, et al. Neuropsychological functioning in bipolar disorder and schizophrenia. Biol Psychiatry (2007) 62:179-86. doi:10.1016/j.biopsych.2006.09.025

18. Seidman LJ, Kremen WS, Koren D, Faraone SV, Goldstein JM, Tsuang MT. A comparative profile analysis of neuropsychological functioning in patients with schizophrenia and bipolar psychoses. Schizophr Res (2002) 53:31-44. doi:10.1016/S0920-9964(01)00162-1

19. Craddock N, Owen MJ. The beginning of the end for the Kraepelinian dichotomy. Br J Psychiatry (2005) 186:364-6. doi:10.1192/bjp.186.5.364

20. Craddock N, Owen MJ. Rethinking psychosis: the disadvantages of a dichotomous classification now outweigh the advantages. World Psychiatry (2007) 6:84-91.

21. Arnone D, Cavanagh J, Gerber D, Lawrie SM, Ebmeier KP, McIntosh AM. Magnetic resonance imaging studies in bipolar disorder and schizophrenia: meta-analysis. Br J Psychiatry (2009) 195:194-201. doi:10.1192/bjp.bp.108. 059717

22. De Peri L, Crescini A, Deste G, Fusar-Poli P, Sacchetti E, Vita A. Brain structural abnormalities at the onset of schizophrenia and bipolar disorder: a metaanalysis of controlled magnetic resonance imaging studies. Curr Pharm Des (2012) 18:486-94. doi:10.2174/138161212799316253

23. Lee KW, Woon PS, Teo YY, Sim K. Genome wide association studies (GWAS) and copy number variation (CNV) studies of the major psychoses: what have we learnt? Neurosci Biobehav Rev (2012) 36:556-71. doi:10.1016/j.neubiorev.2011. 09.001

24. Williams HJ, Norton N, Dwyer S, Moskvina V, Nikolov I, Carroll L, et al. Fine mapping of ZNF804A and genome-wide significant evidence for its involvement in schizophrenia and bipolar disorder. Mol Psychiatry (2011) 16:429-41. doi:10.1038/mp.2010.36

25. Altshuler LL, Ventura J, van Gorp WG, Green MF, Theberge DC, Mintz J. Neurocognitive function in clinically stable men with bipolar I disorder or schizophrenia and normal control subjects. Biol Psychiatry (2004) 56:560-9. doi:10.1016/j.biopsych.2004.08.002

26. Daban C, Martinez-Aran A, Torrent C, Tabarés-Seisdedos R, Balanzá-Martinez $\mathrm{V}$, Salazar-Fraile J, et al. Specificity of cognitive deficits in bipolar disorder versus schizophrenia. Psychother Psychosom (2006) 75:72-84. doi:10.1159/000090891
27. Glahn DC, Bearden CE, Cakir S, Barrett JA, Najt P, Monkul ES, et al. Differential working memory impairment in bipolar disorder and schizophrenia: effect of lifetime history of psychosis. Bipolar Disord (2006) 8:117-23. doi:10.1111/j.1399-5618.2006.00296.x

28. Meesters PD, Schouws S, Stek M, de Haan L, Smit J, Eikelenboom P, et al. Cognitive impairment in late life schizophrenia and bipolar I disorder. Int J Geriatr Psychiatry (2013) 28:82-90. doi:10.1002/gps.3793

29. Sánchez-Morla EM, Barabash A, Martínez-Vizcaíno V, Tabarés-Seisdedos R, Balanzá-Martínez V, Gabranes-Díaz JA, et al. Comparative study of neurocognitive function in euthymic bipolar patients and stabilized schizophrenia patients. Psychiatry Res (2009) 169:220-8. doi:10.1016/j.psychres.2008.06.032

30. Simonsen C, Sundet K, Vaskinn A, Birkenaes AB, Engh JA, Færden A, et al. Neurocognitive dysfunction in bipolar and schizophrenia spectrum disorders depends on history of psychosis rather than diagnostic group. Schizophr Bull (2011) 37:73-83. doi:10.1093/schbul/sbp034

31. Varga M, Magnusson A, Flekkøy K, David AS, Opjordsmoen S. Clinical and neuropsychological correlates of insight in schizophrenia and bipolar I disorder: does diagnosis matter? Compr Psychiatry (2007) 48:583-91. doi:10.1016/j. comppsych.2007.06.003

32. Bora E, Yucel M, Fornito A, Berk M, Pantelis C. Major psychoses with mixed psychotic and mood symptoms: are mixed psychoses associated with different neurobiological markers. Acta Psychiatr Scand (2008) 118:172-87. doi:10.1111/j.1600-0447.2008.01230.x

33. First MB, Spitzer RL, Gibbon M, Williams JBW. Structured Clinical Interview for DSM-IV Axis I Disorders-Patient Version. (SCID-P). Washington, DC: American Psychiatric Press (1994).

34. First MB, Spitzer RL, Gibbon M, Williams JBW. Structured Clinical Interview for DSM-IV Axis I Disorders-non-Patient Version. (SCID-P). Washington, DC: American Psychiatric Press (2002).

35. Keefe RSE, Goldberg TE, Harvey PD, Gold JM, Poe MP, Coughenour L. The brief assessment of cognition in schizophrenia: reliability, sensitivity, and comparison with a standard neurocognitive battery. Schizophr Res (2004) 68:283-97. doi:10.1016/j.schres.2003.09.011

36. Ueoka Y, Tomotake M, Tanaka T, Kaneda Y, Taniguchi K, Nakataki M, et al. Quality of life and cognitive dysfunction in people with schizophrenia. Prog Neuropsychopharmacol Biol Psychiatry (2011) 35:53-9. doi:10.1016/j.pnpbp.2010.08.018

37. Stone MH, Jastak S, Wilkinson G. Wide Range Achievement Test. New York: Harcourt Assessment (1995).

38. American Psychiatric Association. Diagnostic and Statistical Manual of Mental Disorders, Fourth Edition, Text Revision (DSM-IV-TR). Washington, DC: American Psychiatric Association (2000).

39. Kay SR, Fiszbein A, Opler LA. The positive and negative syndrome scale (PANSS) for schizophrenia. Schizophr Bull (1987) 13:261-76. doi:10.1093/schbul/13.2. 261

40. Young RC, Biggs JT, Ziegler VE, Meyer DA. A rating scale for mania: reliability, validity and sensitivity. Br J Psychiatry (1978) 133:429-35. doi:10.1192/bjp.133. 5.429

41. Morice R. Cognitive inflexibility and pre-frontal dysfunction in schizophrenia and mania. Br J Psychiatry (1990) 157:50-4. doi:10.1192/bjp.157.1.50

42. Craddock N, O’Donovan MC, Owen MJ. Genes for schizophrenia and bipolar disorder? Implications for psychiatric nosology. Schizophr Bull (2006) 32:9-16. doi:10.1093/schbul/sbj033

43. Goes FS, Hamshere ML, Seifuddin F, Pirooznia M, Belmonte-Mahon P, Breuer $\mathrm{R}$, et al. Genome-wide association of mood-incongruent psychotic bipolar disorder. Transl Psychiatry (2012) 2:e180. doi:10.1038/tp.2012.106

44. Lichenstein P, Yip BH, Björk C, Pawitan Y, Cannon TD, Sullivan PF, et al. Common genetic determinants of schizophrenia and bipolar disorder in Swedish families: a population-based study. Lancet (2009) 373:234-9. doi:10.1016/ S0140-6736(09)60072-6

45. Purcell SM, Wray NR, Stone JL, Visscher PM, O'Donovan MC, Sullivan PF, et al Common polygenic variation contributes to risk of schizophrenia and bipolar disorder. Nature (2009) 460:748-52. doi:10.1038/nature08185

46. Andreassen OA, Thompson WK, Schork AJ, Ripke S, Mattingsdal M, Kelsoe JR, et al. Improved detection of common variants associated with schizophrenia and bipolar disorder using pleiotropy-informed conditional false discovery rate. PLoS Genet (2013) 9:e1003455. doi:10.1371/journal.pgen.1003455

47. Anderson D, Ardekani BA, Burdick KE, Robinson DG, John M, Malhotra $\mathrm{AK}$, et al. Overlapping and distinct gray and white matter abnormalities 
in schizophrenia and bipolar I disorder. Bipolar Disord (2013) 15:680-93. doi:10.1111/bdi.12096

48. McDonald C, Bullmore ET, Sham PC, Chitnis X, Wickham H, Bramon E, et al. Association of genetic risks for schizophrenia and bipolar disorder with specific and generic brain structural endophenotypes. Arch Gen Psychiatry (2004) 61:974-84. doi:10.1001/archpsyc.61.10.974

49. Schuster C, Schuller AM, Paulos C, Namer I, Pull C, Danion JM, et al. Gray matter volume decreases in elderly patients with schizophrenia: a voxel-based morphometry study. Schizophr Bull (2012) 38:796-802. doi:10.1093/schbul/sbq150

50. Rajji TK, Voineskos AN, Butters MA, Miranda D, Arenovich T, Menon M, et al. Cognitive performance of individuals with schizophrenia across seven decades: a study using the MATRICS Consensus Cognitive Battery. Am J Geriatr Psychiatry (2013) 21:108-18. doi:10.1016/j.jagp.2012.10.011

51. Smith MJ, Barch DM, Csernansky JG. Bridging the gap between schizophrenia and psychotic mood disorders: relating neurocognitive deficits to psychopathology. Schizophr Res (2009) 107:69-75. doi:10.1016/j.schres.2008.07.014

52. Gildengers AG, Chisholm D, Butters MA, Anderson SJ, Begley A, Holm M, et al. Two-year course of cognitive function and instrumental activities of daily living in older adults with bipolar disorder: evidence for neuroprogression? Psychol Med (2013) 43:801-11. doi:10.1017/S0033291712001614

53. Arts B, Jabben N, Krabbendam L, van Os J. A 2-year naturalistic study on cognitive function in bipolar disorder. Acta Psychiatr Scand (2011) 123:190-205. doi:10.1111/j.1600-0447.2010.01601.x

54. Donaldson S, Goldstein LH, Landau S, Raymont V, Frangou S. The Maudsley bipolar disorder project: the effect of medication, family history, and duration of illness on IQ and memory in bipolar I disorder. J Clin Psychiatry (2004) 64:86-93. doi:10.4088/JCP.v64n0116

55. Keefe RSE, Bilder RM, Davis SM, Harvey PD, Palmer BW, Gold JM, et al. Neurocognitive effects of antipsychotic medications in patients with chronic schizophrenia in the CATIE trial. Arch Gen Psychiatry (2007) 64:633-47. doi:10.1001/archpsyc.64.6.633

56. Mishara AL, Goldberg TE. A meta-analysis and critical review of the effects of conventional neuroleptic treatment on cognition in schizophrenia: opening a closed book. Biol Psychiatry (2004) 55:1013-22. doi:10.1016/j.biopsych.2004. 01.027

57. Rossi A, Arduini L, Daneluzzo E, Bustini M, Prosperini P, Stratta P. Cognitive function in euthymic bipolar patients, stabilized schizophrenic patients, and healthy controls. J Psychiatr Res (2000) 34:333-9. doi:10.1016/S0022-3956(00) 00025-X
58. Pardo PJ, Knesevich MA, Vogler GP, Pardo JV, Twone B, Cloninger CR, et al. Genetic and state variables of neurocognitive dysfunction in schizophrenia: a twin study. Schizophr Bull (2000) 26:459-77. doi:10.1093/oxfordjournals. schbul.a033466

59. Sobzcak S, Honig A, Schmitt JAJ, Riedel WJ. Pronounced cognitive deficits following an intravenous L-tryptophan challenge in first-degree relatives of bipolar patients compared to healthy controls. Neuropsychopharmacology (2003) 28:711-9. doi:10.1038/sj.npp.1300055

60. Sarapas C, Shankman SA, Harrow M, Goldberg JF. Parsing trait and state effects of depression severity on neurocognition: evidence from a 26-year longitudinal study. J Abnorm Psychol (2012) 121:830-7. doi:10.1037/a0028141

61. Gopal YV, Variend H. First-episode schizophrenia: review of cognitive deficits and cognitive remediation. Adv Psychiatr Treat (2005) 11:38-44. doi:10.1192/ apt.11.1.38

62. Serrani D. Neurocognitive assessment of ultra high risk of psychosis states using the MATRICS battery (measurement and treatment research to improve cognition in schizophrenia). Rev Psiq Clin (2011) 38:130-4. doi:10.1590/S010160832011000400003

63. American Psychiatric Association. Diagnostic and Statistical Manual of Mental Disorders, Fifth Edition (DSM-V). Arlington, VA: American Psychiatric Publishing (2013).

Conflict of Interest Statement: The authors declare that the research was conducted in the absence of any commercial or financial relationships that could be construed as a potential conflict of interest.

Received: 01 August 2013; accepted: 21 November 2013; published online: 05 December 2013.

Citation: Kuswanto CN, Sum MY and Sim K (2013) Neurocognitive functioning in schizophrenia and bipolar disorder: clarifying concepts of diagnostic dichotomy vs. continuum. Front. Psychiatry 4:162. doi: 10.3389/fpsyt.2013.00162

This article was submitted to Schizophrenia, a section of the journal Frontiers in Psychiatry.

Copyright $(0) 2013$ Kuswanto, Sum and Sim. This is an open-access article distributed under the terms of the Creative Commons Attribution License (CC BY). The use, distribution or reproduction in other forums is permitted, provided the original author(s) or licensor are credited and that the original publication in this journal is cited, in accordance with accepted academic practice. No use, distribution or reproduction is permitted which does not comply with these terms. 